\title{
Crescimento e mortalidade de Oligosarcus hepsetus (Cuvier, 1829) (Characiformes, Characidae) no Parque Estadual da Serra do Mar, Núcleo Santa Virgínia, São Paulo, Brasil
}

\author{
Alberto Luciano Carmassi ${ }^{1}$, Giulianna Rodrigues Rondineli ${ }^{1}$, \\ Leandro Muller Gomiero ${ }^{1,2}$ \& Francisco Manoel de Souza Braga \\ ${ }^{1}$ Departamento de Zoologia, Instituto de Biociências, Universidade Estadual Paulista - UNESP, \\ Av. 24-A, 1515, CP 199, CEP 13506-900, Bela Vista, Rio Claro, SP, Brasil \\ ${ }^{2}$ Autor para correspondência: Leandro Muller Gomiero, e-mail: leanmg@rc.unesp.br
}

CARMASSI, A.L., RONDINELI, G.R., GOMIERO, L.M. \& BRAGA, F.M.S. Growth and mortality of Oligosarcus hepsetus (Cuvier, 1829) (Characiforms, Characidae) in Serra do Mar State Park, Santa Virgínia Unit, São Paulo, Brazil. Biota Neotrop. 11(2): http://www.biotaneotropica.org.br/v11n2/en/ abstract?article+bn00611022011.

\begin{abstract}
Growth and mortality parameters were estimated for Oligosarcus hepsetus caught in the Serra do Mar State Park, Núcleo Santa Virginia. Monthly length frequencies distributions were analyzed with the ELEFAN method within the FISAT program, using the seasonal version of the Von Bertalanffy's growth curve. The instantaneous rate of natural mortality was calculated using the empirical formula proposed by Pauly. The estimated parameters were $\mathrm{K}=0.72, \mathrm{~L} \infty=23.3 \mathrm{~cm}, \mathrm{C}=0.3, \mathrm{Wp}=0.7$ and $\mathrm{M}=1.25$. These parameters corroborate the characteristics of a predator species with long reproductive period, spawning parceled out without parental care, and that lives in a typical river of Atlantic forest.
\end{abstract}

Keywords: dogfish, neotropical freshwater species, population parameters, length frequencies, Atlantic Forest.

CARMASSI, A.L., RONDINELI, G.R., GOMIERO, L.M. \& BRAGA, F.M.S. Crescimento e mortalidade de Oligosarcus hepsetus (Cuvier, 1829) (Characiformes, Characidae) no Parque Estadual da Serra do Mar, Núcleo Santa Virgínia, São Paulo, Brasil. Biota Neotrop. 11(2): http://www.biotaneotropica.org.br/v11n2/pt/ abstract?article+bn00611022011.

Resumo: Parâmetros de crescimento e mortalidade foram estimados para Oligosarcus hepsetus capturados no Parque Estadual da Serra do Mar, Núcleo Santa Virgínia. A distribuição mensal das frequências de comprimento foi analisada pelo método ELEFAN, através do programa FISAT, utilizando-se a versão sazonal da curva de crescimento de Von Bertalanffy. A taxa instantânea de mortalidade natural foi calculada pela fórmula empírica proposta por Pauly. Os parâmetros estimados foram $\mathrm{K}=0,72, \mathrm{~L} \infty=23,3 \mathrm{~cm}, \mathrm{C}=0,3, \mathrm{Wp}=0,7$ e $\mathrm{M}=1,25$. Estes parâmetros corroboram as características de uma espécie predadora com longo período reprodutivo, desova parcelada sem cuidado com a prole e que vive em um rio típico de Mata Atlântica.

Palavras-chave: peixe-cachorro, espécie dulciaquícola neotropical, parâmetros populacionais, frequência de comprimento, Mata Atlântica. 


\section{Introdução}

As estimativas dos parâmetros de crescimento das espécies de peixe são de importância fundamental para o entendimento de eventos do ciclo de vida. A determinação da idade através da contagem de anéis anuais em partes duras, como escamas, otólitos e vértebras é bem conhecida para peixes de regiões temperadas (Ricker 1975), onde flutuações ambientais entre o verão e o inverno são bem nítidas. Em regiões onde as condições climáticas são menos variáveis, como as regiões tropicais e subtropicais, as marcas de aposição são menos definidas e há a necessidade de validar as marcas para que os parâmetros de crescimento obtidos sejam confiáveis e precisos (Ambrósio \& Hayashi 1997, Ambrósio et al. 2003, Feitoza et al. 2004). No entanto, a validação das classes etárias nem sempre é possível, devido principalmente às dificuldades inerentes para a obtenção de número razoável de exemplares ou a pequena definição dos anéis etários (Lizama \& Vazzoler 1993). Apesar de toda a dificuldade encontrada, existem diversos trabalhos que fizeram uso da contagem dos anéis e obtiveram resultados na determinação da idade em peixes de águas tropicais (Barbieri et al. 2001, Feitoza et al. 2004, Penha et al. 2004, Penna et al. 2005, Schulz \& Leal 2005).

Uma alternativa para se estimar os parâmetros de crescimento é a utilização de métodos indiretos, por meio de dados de frequência de comprimento, que são facilmente obtidos junto às populações biológicas. Esses dados contêm informações sobre o comprimento médio em cada grupo etário, juntamente com a posição das modas associadas com as coortes (Basson et al. 1988). Este método foi utilizado para estimar parâmetros populacionais em diversas espécies: Moenkhausia intermedia (Lizama \& Ambrósio 2003), Roeboides paranensis (Capistrano-Santana et al. 2004), Characidium lauroi e C. alipioi (Braga et al. 2007), Moenkhausia dichroura (Cunha et al. 2007), Brycon opalinus (Gomiero et al. 2007) e Cyphocarax modestus (Carmassi et al. 2008).

O gênero Oligosarcus Günther, 1864 compreende 17 espécies válidas, distribuídas pelas principais bacias hidrográficas sul americanas (Ribeiro 2007). Estudos envolvendo aspectos reprodutivos de espécies do gênero são encontrados nos trabalhos de Neves et al. (1995), Hartz et al. (1997), Fialho et al. (1998), Hermes-Silva et al. (2004) e Nunes et al. (2004). A dieta foi investigada por Hartz et al. (1996) e Nunes \& Hartz (2006); e o crescimento de Oligosarcus jenynsi por análise e contagem dos anéis etários Hartz \& Barbieri (1995).

Oligosarcus hepsetus é um Characiforme carnívoro de pequeno a médio porte (Géry 1977) com ampla distribuição nos ambientes de água doce do sudeste do Brasil (Santos et al. 2005, 2006). Na literatura, encontram-se disponíveis informações sobre a dieta (Araújo et al. 2005) e o desenvolvimento ovariano e testicular dessa espécie no reservatório de Lajes - RJ (Santos et al. 2005, 2006). No Núcleo Santa Virgínia, onde a espécie não é explorada pela pesca, informações sobre a biologia alimentar e reprodutiva dessa espécie foram recentemente obtidas. A alimentação com maior intensidade no verão e no outono apresentou diferenciação ontogenética sendo os indivíduos menores (5 a $20 \mathrm{~cm}$ ) principalmente insetívoros e os maiores $(>20 \mathrm{~cm}$ ) restritamente piscívoros (Botelho et al. 2007). $\mathrm{O}$ período reprodutivo foi longo ( 8 meses), porém mais intenso nos períodos com mais chuvas. A desova foi do tipo parcelada sem cuidado parental, a fecundidade foi relativamente baixa quando comparada às espécies reofílicas e a maturação gonadal foi precoce quando se compara a mesma espécie em outras localidades (Gomiero et al. 2008).

Como as informações sobre o crescimento e a mortalidade são essenciais para o manejo das populações de peixes (Froese \& Binohlan 2000), o presente trabalho teve como objetivo estimar os parâmetros de crescimento, bem como coeficientes de mortalidade para Oligosarcus hepsetus através de dados de frequência de comprimento no Núcleo Santa Virgínia, Parque Estadual da Serra do Mar.

\section{Material e Métodos}

Os exemplares foram capturados em doze amostras mensais realizadas durante o ano de 2004, no rio Grande, que pertence à bacia do rio Paraíba do Sul e corre no interior do Núcleo Santa Virgínia, no

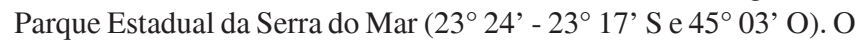
clima da região é tropical temperado, de acordo com a classificação climática de Köeppen (Setzer 1966 apud Tabarelli \& Mantovani 1999). A precipitação média anual é de 2,180 mm, considerada uma das mais elevadas do estado de São Paulo, sendo dezembro, janeiro e fevereiro os meses mais chuvosos, e junho, julho e agosto, os menos chuvosos (Tabarelli \& Mantovani 1999).

Os peixes foram capturados com o uso de seis redes-de-espera (cada unidade com $10 \mathrm{~m}$ de comprimento e 1,5 m de altura e malhagens de 1,$5 ; 2,0 ; 2,5 ; 3,0 ; 3,5$ e 4,0 cm entre nós adjacentes), peneira e armadilhas do tipo covo, fixados em formalina $10 \%$ e conservados em álcool $70 \%$ até a realização das análises. Todas as amostragens foram padronizadas quanto as metragens, as malhagens das redes, a quantidade de covos (três) e a quantidade de passagens da peneira. No laboratório, de cada exemplar foram obtidos dados de comprimento total (cm) e sexo (Braga 1990).

Exemplares de Oligosarcus hepsetus foram depositados no Laboratório de Ictiologia do Departamento de Zoologia da Universidade Estadual Paulista (UNESP) em Rio Claro, São Paulo.

Os parâmetros de crescimento foram estimados pelo método ELEFAN I (Eletronic Lengths - Frequency Analysis) e para a sua aplicação utilizou-se o programa FISAT (FAO - ICLARM Stock Assessment Tools) (Gayanilo Junior et al. 1996, Gayanilo Junior \& Pauly 1997). Esse programa se fundamenta no deslocamento modal de sequências temporais de amostras de comprimento (Castro et al. 2002), utilizando a versão sazonal da curva de crescimento de Von Bertalanffy (Sparre \& Venema 1997), cujo modelo é (Equação 1):

$\mathrm{L}_{\mathrm{t}}=\mathrm{L} \infty \times\left[1-\exp \left\{\left(-\mathrm{K} \times\left(\mathrm{t}-\mathrm{t}_{0}\right)-(\mathrm{CK} / 2 \pi) \times \operatorname{seno}\left(2 \pi \times\left(\mathrm{t}-\mathrm{t}_{\mathrm{S}}\right)\right)\right\}\right](1)\right.$ em que:

$\mathrm{L}_{\mathrm{t}}$ é o comprimento na idade $\mathrm{t}, \pi$ equivale a 3,14159 e $\mathrm{t}_{\mathrm{s}}$ é a época do ano de maior taxa de crescimento, podendo ser calculado através da expressão $\mathrm{t}_{\mathrm{s}}=0,5+\mathrm{Wp}$. Assim, o modelo utilizado considera, além dos parâmetros L $\infty$ (comprimento assintótico) e K (taxa de crescimento), outros dois parâmetros: Wp ("winter point") e C (amplitude de oscilação sazonal). O valor de C está relacionado à diferença de temperaturas superficiais médias da água de inverno e verão, variando conforme a região de estudo; e Wp representa a época do ano em que o crescimento é mais lento, normalmente o período mais frio, sendo uma relação do número de dias até o mês de menor crescimento da espécie com o número de dias do ano (Pauly \& Gaschutz 1979, Castro et al. 2002).

A taxa instantânea de mortalidade natural (M) foi estimada utilizando-se os parâmetros: taxa de crescimento $(\mathrm{K})$, comprimento assintótico $(\mathrm{L} \infty)$ e temperatura média da água em graus centígrados no período, segundo a Equação 2 empírica proposta por Pauly (1980), sendo:

$\log _{10} \mathrm{M}=-0,0066-0,279 \times \log _{10} \mathrm{~L} \infty+\log _{10} \mathrm{k}+0,4634 \times \log _{10} \mathrm{~T}^{\circ}$

Apesar de Oligosarcus hepsetus apresentar desova do tipo parcelada, houve um pico reprodutivo (Gomiero et al. 2008), o que tornou possível a identificação de diferentes coortes nas distribuições 
de frequência de comprimento, condição essencial para que esse tipo de análise seja conduzida (Sparre \& Venema 1997).

Foram registradas, para cada amostragem, medidas de temperatura da água $\left({ }^{\circ} \mathrm{C}\right)$.

\section{Resultados}

As distribuições de frequências numéricas de indivíduos por mês, ao longo do período de estudo está na Figura 1.

Foram utilizados dados de comprimento total de 117 indivíduos de Oligosarcus hepsetus. Destes exemplares, 44 eram fêmeas, 52 machos e 21 imaturos. O menor indivíduo capturado apresentou $4,2 \mathrm{~cm}$ (imaturo) e o maior $22 \mathrm{~cm}$ (fêmea) (Figura 2).

As estimativas dos parâmetros de crescimento a partir dos dados de frequência de comprimento foram: $\mathrm{K}=0,72 /$ ano e $\mathrm{L} \infty=23,3 \mathrm{~cm}$. O estimador da amplitude de oscilação sazonal (C) foi de 0,3 , indicando uma diferença de $3{ }^{\circ} \mathrm{C}$ entre as temperaturas superficiais médias da água no período. $\mathrm{O}$ ponto de inverno $(\mathrm{Wp}$ ) foi de 0,7 mostrando que a época de menor crescimento ocorreu a partir de agosto. A curva de crescimento e as coortes obtidas para Oligosarcus hepsetus através do método ELEFAN I encontram-se na Figura 3. Seis coortes podem se observadas, sendo a primeira formada no mês de abril (coincidindo com o final do período reprodutivo) quando se inicia o primeiro ano de vida.

A taxa instantânea de mortalidade total (Z) foi considerada igual à taxa instantânea de mortalidade natural (M) (Pauly 1998). Considerando a temperatura média da água no período de estudo de $17,9^{\circ} \mathrm{C}$, a taxa instantânea de mortalidade natural foi de 1,25.

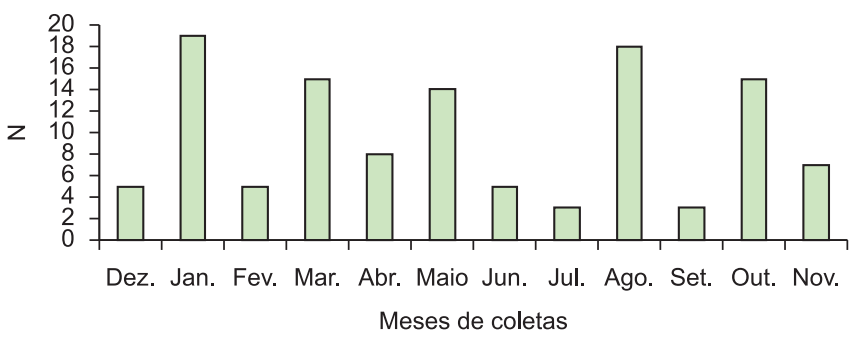

Figura 1. Distribuição de frequência mensal dos exemplares de Oligosarcus hepsetus do Parque Estadual da Serra do Mar, Núcleo Santa Virgínia, São Paulo, Brasil, em 2004.

Figure 1. Frequencies distributions monthly for Oligosarcus hepsetus, from Parque Estadual da Serra do Mar, Santa Virgínia complex, São Paulo, Brasil, 2004.

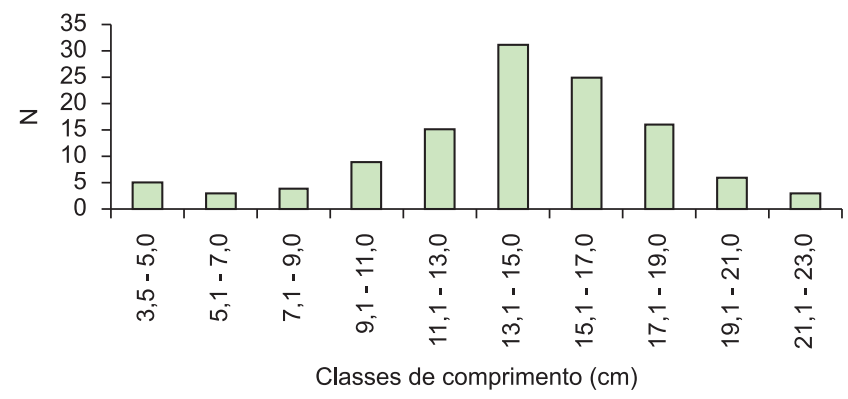

Figura 2. Distribuição de frequência de classes de comprimento total de Oligosarcus hepsetus do Parque Estadual da Serra do Mar, Núcleo Santa Virgínia, São Paulo, Brasil, em 2004.

Figure 2. Total length frequencies distributions for Oligosarcus hepsetus, from Parque Estadual da Serra do Mar, Santa Virgínia complex, São Paulo, Brasil, 2004.

\section{Discussão}

De acordo com Beverton \& Holt (1957), L $\infty$ é afetado por fatores como suprimento alimentar e densidade populacional e $\mathrm{K}$ é determinado genética e/ou fisiologicamente, sendo, geralmente, inverso ao valor do comprimento assintótico e ao da longevidade. Em peixes tropicais, Pauly (1998) constatou que elevados valores de K e baixos valores de comprimento assintótico são comuns, e atribui a esse fato a combinação de vários fatores, sendo a temperatura o fator mais importante. Por se tratar de uma espécie piscívora (Géry 1977), o rápido crescimento é determinante contra a predação que pode ser iniciada ainda na fase de larvas (Schütz et al. 2008) e para a melhor obtenção de alimento (Gerking 1994).

Oligosarcus hepsetus apresentou alta taxa de crescimento $(\mathrm{K}=0,71)$ e um valor relativamente baixo de comprimento assintótico $(\mathrm{L} \infty=23,3)$. Oligosarcus jenynsii, na lagoa do Caconde (RS), apresentou valores mais baixos na taxa de crescimento: $K=0,608$ para machos e $\mathrm{K}=0,316$ para fêmeas; apesar de os machos crescerem mais rápido do que as fêmeas, estas foram mais longevas, atingindo maiores comprimentos assintóticos $(\mathrm{L} \infty=25,8 \mathrm{~cm}$ para fêmeas e $\mathrm{L} \infty=20,4 \mathrm{~cm}$ para machos) (Hartz \& Barbieri 1995). $\mathrm{Na}$ área de estudo, as fêmeas de $O$. hepsetus também atingiram maiores comprimentos do que os machos (Gomiero et al. 2008). Esta característica parece ser comum para as espécies de peixes tropicais devido principalmente à garantia de maior fecundidade (Lowe-McConnell 1999). A estimativa do comprimento assintótico de $O$. hepsetus parece precisa, já que ao utilizar outro método de estimativa, como o proposto por Pauly (1983), um valor muito próximo pode ser encontrado $\left(\mathrm{L} \infty=\mathrm{L}_{\text {máx }} / 0,95=22 / 0.95=23,15\right)$.

Os peixes tropicais tendem a ter, para qualquer tamanho assintótico ou taxa de crescimento, altos valores de mortalidade natural (Pauly 1998). Oligosarcus hepsetus apresentou alta mortalidade (1,25), maior do que Brycon opalinus, com ocorrência na mesma área, e Cyphocarax modestus, no Ribeirão Claro, que apresentaram taxas instantâneas de mortalidade natural próximas a 1,0 (Gomiero et al. 2007, Carmassi et al. 2008). Vazzoler (1996) aponta que o período reprodutivo das espécies piscívoras geralmente antecede o das demais espécies do sistema, provavelmente para que suas larvas já estejam mais desenvolvidas e tenham maior possibilidade de predar as larvas das demais espécies quando estas eclodirem. Por um lado, as desovas precoces garantem o alimento para os juvenis, porém, por outro podem aumentar a mortalidade de ovos e larvas devido às baixas temperaturas e demais condições adversas (Fialho et al. 1998). Oligosarcus jenynsii, na lagoa da Custódia (RS) (Fialho et al. 1998)

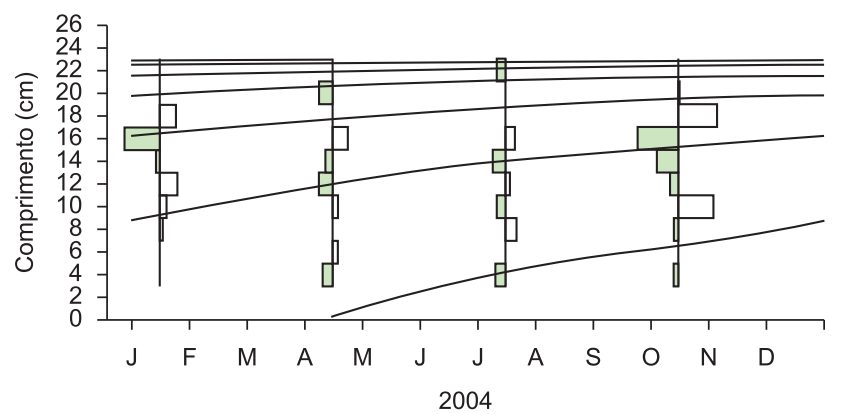

Figura 3. Curva de crescimento de Oligosarcus hepsetus reestruturada para frequência de comprimento total, calculada por meio do sistema ELEFAN I (pacote computacional FISAT), do Parque Estadual da Serra do Mar, Núcleo Santa Virgínia, São Paulo, Brasil, em 2004.

Figure 3. Growth curves of Oligosarcus hepsetus restructured for frequency of total length, estimated through the ELEFAN I (FISAT software) from Parque Estaudal da Serra do Mar, Santa Virgínia complex, São Paulo, Brasil, 2004. 
e O. hepsetus, no Núcleo Santa Virgínia (Gomiero et al. 2008) apresentaram período reprodutivo anterior ao das demais espécies, indo de encontro com as informações apresentadas acima. Assim, à alta taxa de mortalidade encontrada pra $O$. hepsetus poder ser explicada, em parte, pela elevada mortalidade nas fases iniciais de desenvolvimento. Por outro lado, a presença de potenciais predadores no sistema e até mesmo a ocorrência de canibalismo também podem contribuir para um aumento na taxa de mortalidade, embora nenhuma evidência tenha sido observada.

A época de menor crescimento se deu a partir de agosto, período com a mais baixa temperatura na região, registrada junto à Estação Metereológica do Núcleo Santa Virgínia (Souza 2009), o que indica que a redução do crescimento de $O$. hepsetus está relacionada com o decréscimo da temperatura. Além disso, a época de menor crescimento coincidiu com o início do período reprodutivo (Gomiero et al. 2008) e a menor intensidade da atividade alimentar (Botelho et al. 2007), assim, a energia armazenada investida no crescimento somático passa a ser direcionada para a maturação dos gametas. Nunes \& Hartz (2006) verificaram que outras duas espécies de Oligosarcus (O. jenynsii e $O$. robustus) deixaram de alocar energia para o fígado durante o período reprodutivo.

O aspecto polimodal da distribuição de frequência de comprimento, com a presença de seis coortes, é compatível com a informação de tratar-se de uma população bem estabelecida no ambiente (Cunha et al. 2007). O recrutamento, que é a entrada de novos indivíduos na população, ocorre entre abril, maio e outubro, coincidindo com o fim do período reprodutivo da espécie (Gomiero et al. 2008). Nunes et al. (2004) também encontraram coincidência entre o recrutamento e o final da estação de desova para $O$. jenynsii e $O$. robustus.

O crescimento e a mortalidade de Oligosarcus hepsetus corroboram as características de uma espécie predadora com longo período reprodutivo, desova parcelada sem cuidado com a prole e que vive em um rio típico de Mata Atlântica.

\section{Agradecimentos}

À FAPESP (03/05696-1) pela bolsa de pós-doutorado, à Cotec (40.673/03) e ao IBAMA (055/2003).

\section{Referências bibliográficas}

AMBRósio, A.M., GOMES, L.C. \& AGOSTINHO, A.A. 2003. Age and growth of Hypophthalmus edentatus (Spix), (Siluriformes, Hypophthalmidae) in the Itaipu Reservoir, Paraná, Brazil. Rev. Bras. Zool. 20(2):183-190.

AMBRÓSIO, A.M. \& HAYASHI, C. 1997. Idade e crescimento de Steindachnerina insculpta (Fernández-Yepes, 1948), (Characiformes, Curimatidae) da planície de inundação do alto Rio Paraná, Brasil. Rev. Bras. Biol. 57(4):687-698.

ARAÚJO, F.G., ANDRADE, C.C., SANTOS, R.N., SANTOS, A.F.G.N. \& SANTOS, L.N. 2005. Spatial and seasonal changes in the diet of Oligosarcus hepsetus (Characiformes, Characidae) in a brazilian reservoir. Rev. Bras. Zool. 65(1):1-8.

BARBIERI, G., SALLES, F.A. \& CESTAROLLI, M.A. 2001. Growth and first sexual maturation size of Salminus maxillosus Valenciennes, 1849 (Characiformes, Characidae) in Mogi Guaçu River, State of São Paulo, Brazil. Acta. Sci. Biol. Sci. 23(2):453-459.

BASSON, M., ROSENBERG, A.A. \& BEDDINGTON, J.R. 1988. The accuracy and reliability of two new methods for estimating growth parameters from length-frequency data. J. Cons. Int. Explor. Mer. 44:277-285.

BEVERTON, R.J.H. \& HOLT, S.J. 1957. On the dynamics of exploited fish populations. Fishery Invest. 2(19):1-533.
BOTELHO, M.L.L.A., GOMIERO, L.M. \& BRAGA, F.M.S. 2007. Feeding of Oligosarcus hepsetus (Cuvier, 1829) (Characiformes) in the Serra do Mar State Park - Santa Virgínia Unit, São Paulo, Brazil. Braz. J. Biol. 67(4):741-748. http://dx.doi.org/10.1590/S1519-69842007000400022

BRAGA, F.M.S. 1990. Aspectos da reprodução e alimentação de peixes comuns em um trecho do rio Tocantins entre Imperatriz e Estreito, Estado do Maranhão e Tocantins, Brasil. Rev. Bras. Biol. 50(3):547-558.

BRAGA, F.M.S., SOUZA, U.P. \& CARMASSI, A.L. 2007. Dinâmica populacional de Characidium lauroi e C. alipioi (Teleostei, Crenuchidae) na microbacia do Ribeirão Grande, serra da Mantiqueira oriental (SP). Acta. Sci. Biol. Sci. 29(3):281-287.

CAPISTRANO-SANTANA, A., ALMEIDA, R.S., ÁVILA, R.W. \& CATELLA, A.C. 2004. Estimativa dos parâmetros de crescimento de Roeboides paranensis: diferentes respostas para uma mesma pergunta. In IV Simpósio sobre Recursos Naturais e Sócio-Econômicos do Pantanal. Corumbá.

CARMASSI, A.L., SILVA, A.T., RONDINELI, G.R. \& BRAGA, F.M.S. 2008. Biologia populacional de Cyphocarax modestus (Osteichthyes, Curimatidae) no córrego Ribeirão Claro, município de Rio Claro (SP). Biota Neotrop. 8(1):110-114.

CASTRO, P.M.G., CERGOLE, M.C., CARNEIRO, M.H., MUCINHATO, C.M.D. \& SERVO, G.J.M. 2002. Crescimento, mortalidade e taxa de explotação do goete, Cynoscion jamaicensis (Perciformes: Sciaenidae), na região sudeste/sul do Brasil. B. Inst. Pesca. 28(2):141-153.

CUNHA, N.L., CATEllA, A.C. \& KINAS, M.A. 2007. Growth parameters estimates for a small fish of the Pantanal, Brazil: Moenkhausia dichroura (Characiformes; Characidae). Braz. J. Biol. 67(2):293-297. http://dx.doi.org/10.1590/S1519-69842007000200014

FEITOZA, L.A., OKADA, E.K. \& AMBRÓSIO, A.M. 2004. Idade e crescimento de Pterodoras granulosus (Valenciennes, 1833) (Siluriformes, Doradidae) no reservatório de Itaipu, Estado do Paraná, Brasil. Acta. Sci. Biol. Sci. 26(1):47-53.

FIALHO, C.B., SCHIFINO, L.C. \& VERANI, J.R. 1998. Biologia reprodutiva de Oligosarcus jenynsii (Günther) (Characiformes, Characidae) da lagoa das Custódias, Tramandaí, Rio Grande do Sul, Brasil. Rev. Bras. Zool. 15(3):775-782. http://dx.doi.org/10.1590/S0101-81751998000300020

FROESE, R. \& BINOHLAN, C. 2000. Empirical relationships to estimate asymptotic length, length at first maturity and length at maximum yield per recruit frequency data. J. Fish Biol. 56:758-773. http://dx.doi.org/10.1111/j.1095-8649.2000.tb00870.x

GAYANILO JUNIOR, F.C., SPARRE, P. \& PAULY, D. 1996. The FAOICLARM Stock Assessment Tools (FISAT) User's guide. FAO Comput. Inf. Ser. Fish. 6:1-186.

GAYANILO JUNIOR., F.C. \& PAULY, D. 1997. FAO-ICLARM Stock Assessment Tools (FISAT) Reference Manual. FAO Comput. Inf. Ser. Fish. 8:1-262.

GERKING, S.D. 1994. Feeding ecology of fishes. Academic Press.

GÉRY, J. 1977. Characoids of the world. T.F.H. Publ., Neptune City, USA.

GOMIERO, L.M., CARMASSI, A.L. \& BRAGA, F.M.S. 2007. Crescimento e mortalidade de Brycon opalinus (Characiformes, Characidae) no Parque Estadual da Serra do Mar, Mata Atlântica, Estado de São Paulo. Biota Neotrop. 7(1):22-26. http://dx.doi.org/10.1590/S1519-69842008000100027

GOMIERO, L.M., GARUANA, L. \& BRAGA, F.M.S. 2008. Reproduction of Oligosarcus hepsetus (Cuvier, 1829) (Characiforms) in the Serra do Mar State Park, São Paulo, Brazil. Braz. J. Biol. 68(1):187-192.

HARTZ, S.M. \& BARBIERI, G. 1995. Crescimento do peixe-cachorro Oligosarcus jenynsii (Günther, 1864) na lagoa Caconde, Rio Grande do Sul, Brasil (Teleostei, Chatacidae). B. Inst. Pesca. 22(2):33-40.

HARTZ, S.M., MARTINS, A. \& BARBIERI, G. 1996. Dinâmica da alimentação e dieta de Oligosarcus jenynsii (Günther, 1864) na lagoa Caconde, Rio Grande do Sul, Brasil (Teleostei, Characidae). B. Inst. Pesca. 23:21-29. 
HARTZ, S.M., VILELLA, F.S. \& BARBIERI, G. 1997. Reproduction dynamics of Oligosarcus jenynsii (Characiformes, Characidae) in Lake Caconde, Rio Grande do Sul, Brasil. Rev. Bras. Biol. 57(2):295-303.

HERMES-SILVA, S., MEURER, S. \& ZANIBONI-FILHO, E. 2004 Biologia alimentar e reprodutiva do peixe-cachorro (Oligosarcus jenynsii Günther, 1864) na região do alto rio Uruguai - Brasil. Acta. Sci. Biol. Sci. 26(2):175-179.

LIZAMA, M.A.P. \& AMBRÓSIO, A.M. 2003. Crescimento, recrutamento e mortalidade do pequi Moenkhausia intermedia (Osteichthyes, Characidae) na planície de inundação do alto rio Paraná, Brasil. Acta Sci. Biol. Sci. 25(2):329-333.

LIZAMA, M.A.P. \& VAZZOLER, A.E.A.M. 1993. Crescimento em peixes do Brasil: uma síntese comentada. Rev. UNIMAR. 15(Sup.):141-173.

LOWE-McCONNELL, R.H. 1999. Estudos ecológicos de comunidades de peixes tropicais. (A.E.A.M. Vazzoler, A.A. Agostinho \& P. Cunnighan, trad.). Ed. da Universidade de São Paulo, São Paulo.

NEVES, C.A., ANDRADE, D.R., MATTA, S.L.P., VIDAL, M.V.\& SANTOS, A.A. 1995. Cytochemical analysis of polysaccharides from the cortical alveoli of the oocytes of the lambari-bocarra (Oligosarcus argenteus Günther, 1864) (Pisces, Characidae). Rev. Bras. Biol. 55(4):693-696.

NUNES, D.M. \& HARTZ, S.M. 2006. Feeding dynamics and ecomorphology of Oligosarcus jenynsii (Günther, 1864) and Oligosarcus robustus (Menezes, 1969) in the Lagoa Fortaleza, southern Brazil. Braz. J. Biol. 66(1A):121-132

NUNES, D.M., PELLANDA, M. \& HARTZ, S.M. 2004. Dinâmica reprodutiva de Oligosarcus jenynsii e O. robustus (Characiformes, Characidae) na Lagoa Fortaleza, Rio Grande do Sul, Brasil. Iheringia Sér. Zool. 94(1):5-11. http://dx.doi.org/10.1590/S1519-69842006000100016

PAULY, D. 1980. On the interrelationships between natural mortality, growth parameters, and environmental temperature in 175 fish stocks. ICES. 39(2):175-192.

PAULY, D. 1998. Tropical fishes: patterns and propensities. J. Fish Biol. 53:1-17.

PAULY, D. \& GASCHUTZ, G. 1979. A simple method for fitting oscillating length growth data, with a program for pocket calculator. International Council for the Exploration of the Sea. Council Meeting 1979/G. Demersal Fish Committee.

PENHA, J.M.F., MATEUS, L.A.F. \& BARBIERI, G. 2004. Age and growth of the duckbill catfish (Sorubim cf. lima) in the Pantanal. Braz. J. Biol. 64(1):125-134. http://dx.doi.org/10.1590/S1519-69842004000100014
PENNA, M.A.H., VILLACORTA-CORRÊA, M.A., WALTER, T. \& PETRERE-JR., M. 2005. Growth of the Tambaqui Colossoma macropomum (Cuvier) (Characiformes: Characidae): Which is the best model? Braz. J. Biol. 65(1):129-139.

RIBEIRO, A.C. 2007. Filogenia e biogeografia do gênero Oligosarcus Günther, 1864 (Ostariophysi; Characidae). Tese de Doutorado, Universidade Estadual Paulista, Botucatu.

RICKER, W.E. 1975. Computation and interpretation of biological statistics of fish populations. Bull. Fish. Res. Board. Can. 191:1-382.

SANTOS, R.N., ANDRADE, C.C., SANTOS, A.F.G.N., SANTOS, L.N. \& ARAÚJO, F.G. 2005. Hystological analysis of ovarian development of the Characiform Oligosarcus hepsetus (Cuvier, 1829) in a brazilian reservoir. Braz. J. Biol. 65(1):169-177. http://dx.doi.org/10.1590/S151969842005000100020

SANTOS, R.N., ANDRADE, C.C., SANTOS, L.N., SANTOS, A.F.G.N. \& ARAÚJO, F.G. 2006. Testicular maturation of Oligosarcus hepsetus (Cuvier) (Actinopterygii, Characidae) in a brazilian tropical reservoir Braz. J. Biol. 66(1A):143-150. http://dx.doi.org/10.1590/S151969842006000100018

SCHULZ, U.H. \& LEAL, M.E. 2005. Growth and mortality of Black bass, Micropterus salmoides (Pisces, Centrarchidae; Lacepède, 1802) in a reservoir in southern Brazil. Braz. J. Biol. 65(2):363-369.

SCHÜTZ, J.H., WEINGARTNER, M., ZANIBONI-FILHO, E. \& NUÑER, A.P.O. 2008. Crescimento e sobrevivência de larvas de suruvi Steindachneridion scriptum nos primeiros dias de vida: influência de diferentes alimentos e fotoperíodos. B. Inst. Pesca 34(3):443-451. http://dx.doi.org/10.1590/S1519-69842005000200021

SOUZA, U.P. 2009. Biologia e ciclo de vida de Astyanax cf. scabripinnis paranae Eigenmann, 1914 (Characidae, Tetragonopterinae), no Ribeirão Grande, Parque Estadual da Serra do Mar, Núcleo Santa Virgínia, SP. Tese de Doutorado, Universidade Estadual Paulista, Rio Claro.

SPARRE, P. \& VENEMA, S.C. 1997. Introdução à avaliação de mananciais de peixes tropicais. Parte 1: Manual. FAO, Roma.

TABARELLI, M. \& MANTOVANI, W. 1999. Clareiras naturais e a riqueza de espécies pioneiras em uma floresta atlântica montana. Rev. Brasil. Biol. 59(2):251-261.

VAZZOLER, A.E.A.M. 1996. Biologia da reprodução de peixes teleósteos: Teoria e Prática. Nupelia, Eduem, Maringá. 